\title{
Focused-Ion-Beam Processing for Photonics
}

\author{
René M. de Ridder, Wico C.L. Hopman, and Feridun Ay \\ University of Twente, MESA + Institute for Nanotechnology \\ P.O. Box 217, 7500 AE Enschede, The Netherlands \\ e-mail:R.M.deRidder@utwente.nl
}

\begin{abstract}
Although focused ion beam (FIB) processing is a well-developed technology for many applications in electronics and physics, it has found limited application to photonics. Due to its very high spatial resolution in the order of $10 \mathrm{~nm}$, and its ability to mill almost any material, it seems to have a good potential for fabricating or modifying nanophotonic structures such as photonic crystals. The two main issues are FIB-induced optical loss, e.g., due to implantation of gallium ions, and the definition of vertical sidewalls, which is affected by redeposition effects. The severity of the loss problem was found to depend on the base material, silicon being rather sensitive to this effect. The optical loss can be significantly reduced by annealing the processed samples. Changing the scanning strategy for the ion beam can both reduce the impact of gallium implantation and the redeposition effect.
\end{abstract}

Keywords: focused ion beam, FIB, scanning strategy, damage, gallium implantation, optical loss, photonic crystal.

\section{INTRODUCTION}

Focused ion beam (FIB) processing for nanofabrication has been studied since quite some time, e.g., [1]. Overviews of FIB processing and its applications can be found in recent review papers [2]-[5]. Important "conventional" applications include mask repair, repair or modification of electronic integrated circuits, and sample preparation for transmission electron microscopy. One of the most attractive features of FIB milling is its ability to process hard materials such as $\mathrm{SiC}$ or $\mathrm{LiNbO}_{3}$ which are extremely hard to etch otherwise. The operating modes of FIB machines include ion beam milling (sputtering), ion implantation, ion-induced deposition, and ion-assisted etching. Several of these effects may occur simultaneously, e.g., some ion implantation during a milling process is unavoidable. In addition, unwanted effects like redeposition and amorphization of crystalline materials may limit the attainable accuracy or modify material properties in unacceptable ways. Also, proximity effects due to ion scattering and redeposition may affect the accuracy of FIB-fabricated nanostructures.

The application of FIB to the fabrication of optical micro- and nanostructures, such as photonic crystals, has been reported mainly for III-V gallium compounds, e.g., [6]. Interesting work was also reported on $\mathrm{LiNbO}_{3}$, e.g., [7], and metals, e.g., [8]. Silicon is a very interesting material for nano-optical structures such as photonic crystals, operating in the near infrared. FIB-fabricated photonic structures have been reported [9]-[11] but FIBinduced optical damage is a problem [12]-[15]. Both implantation effects of the gallium ions mostly used for FIB milling, and amorphization may cause severe optical losses; the exact loss mechanism is not yet known. The amorphization effect in silicon has even been exploited for obtaining optical contrast in a proposed ion-beam written optical memory [16].

We have investigated several options for improving the optical quality of FIB-fabricated structures in silicon. Choosing a suitable scanning strategy helps to improve the geometrical quality (reducing redeposition effects leading to steeper sidewalls) and to reduce optical absorption loss (reducing exposure of unmilled regions to the gallium beam). Annealing the FIB-milled samples reduces optical absorption loss by out-diffusion and evaporation of implanted gallium and repair of the damaged crystal lattice. Another method for improving sidewall steepness and reducing implantation damage is to use a hard mask that prevents damage to the remaining silicon due to the spill-over from the tails of the gallium beam, and unwanted etching due to beam movement from one hole location to the next [13]. Another advantage of this method is that material redeposited outside the holes is removed by etching away the hard mask. A step further towards minimising the gallium beam damage to the silicon is to use FIB as a lithography tool only for structuring the hard mask, and fabricating the holes in silicon by reactive ion etching. Finally, in a different vein, one could use a FIB-milled silicon structure as a template for use with, e.g., nano-imprint lithography (NIL), as suggested in [11]. In this case, only the geometrical quality is important, and implantation damage will not affect the optical quality of the final structures that are fabricated using NIL.

In this paper we will report our results on improving the scanning strategy and we will show some preliminary results of annealing structures with FIB-induced implantation damage. All experiments were performed on a FEI Nova 600 dual beam FIB machine with a liquid metal $\mathrm{Ga}^{+}$ion source, using an acceleration voltage of $30 \mathrm{kV}$.

This research was supported by NanoNed, a national nanotechnology program coordinated by the Dutch ministry of Economic Affairs, and was also supported by the European Network of Excellence ePIXnet. 


\section{SCANNING STRATEGY}

Two different scanning strategies have been compared, viz., a conventional raster scan and a method where each individual hole is scanned using a spiral pattern, see Fig. 1.

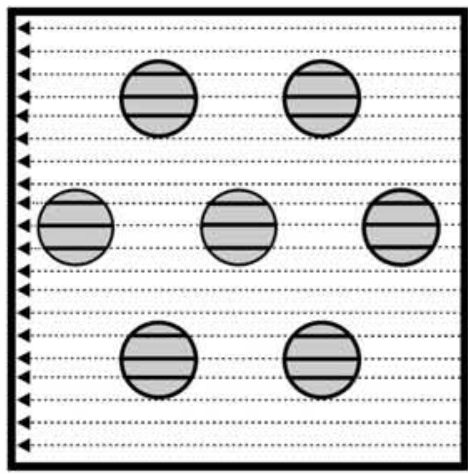

a)

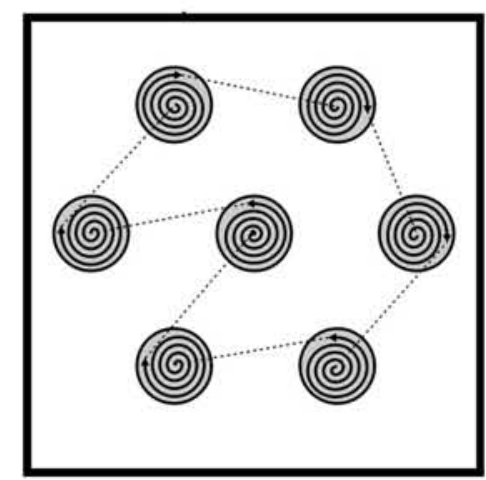

b)

Figure 1. Scanning strategies: a) Raster scan; b) Spiral scan.

The ion beam is not completely suppressed in the nominally unmilled regions; while the beam passes quickly over the regions between holes, a small dose of gallium is implanted, which can have a strong effect on optical absorption. From the figure it is clear that the unwanted beam exposure is much smaller in the case of the spiral scan. Another drawback of the raster pattern is that the beam is always moving in the same direction while milling (from right to left in Fig. 1a). This leads to an asymmetric shape of the holes, as demonstrated in Fig. 2a, while the shape produced by the spiral scan (Fig. 2b) is much more symmetric.

The spiral pattern has another beneficial effect, somewhat improving the sidewall steepness, because redeposited material is removed from the sidewalls as the beam progresses from the centre of a hole outward. The average deviation from verticality of the left and right sidewall, measured halfway the depth of the hole was $19^{\circ}$ for raster scan and $13^{\circ}$ for the spiral scan in bulk silicon, with the milling parameters specified in the caption of Fig. 2. Near the bottom of the raster scanned holes, the sidewall angle is much worse. These deviation angles were calculated from the cross-sections in Fig. 2, taking into account that the images appear to be compressed in the vertical direction due to the angle at which the images were taken.

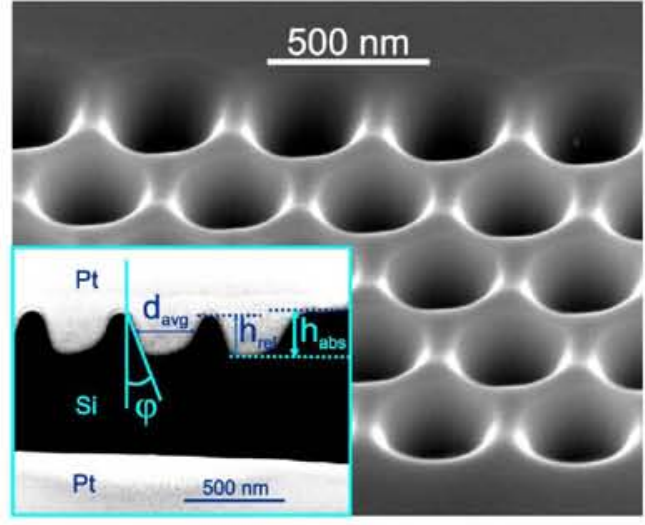

a)

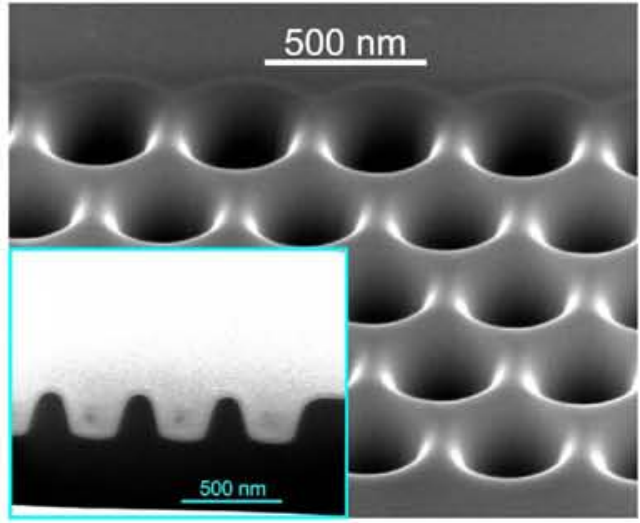

b)

Figure 2. SEM images, comparing $250 \mathrm{~nm}$ diameter holes in a triangular lattice with $440 \mathrm{~nm}$ pitch, milled into bulk silicon using different scanning strategies. a) Raster scan; b) Spiral scan. The insets show cross-sections, where platimum was locally deposited before cross-sectioning with FIB, in order to prevent redeposition artefacts caused by the cross-sectioning cut, and to improve image contrast. Both patterns have been milled using approximately the same dose of $70 \mathrm{pC}$ per hole, with $48 \mathrm{pA}$ ion beam current ( $e^{-1}$ spot diameter $\sim 18 \mathrm{~nm}$ ), $0.1 \mathrm{~ms}$ dwell time per pixel, and repeating the pattern in 12 loops. Both the "top-view" images and the cross-sections were taken at $52^{\circ}$ from the surface normal. 


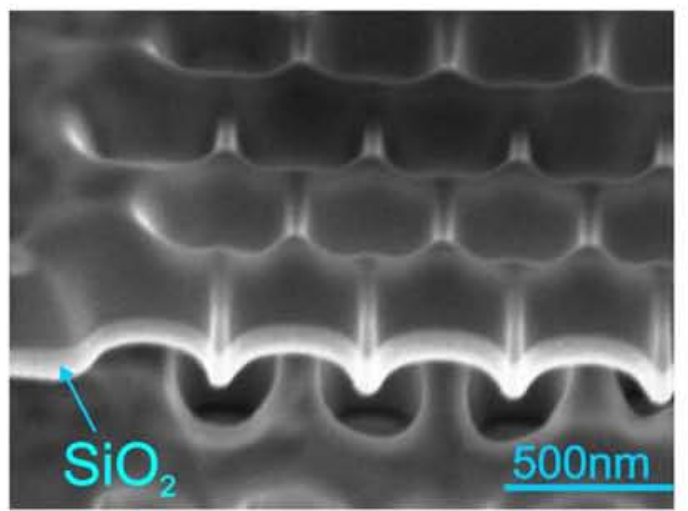

Figure 3. Holes milled into a $220 \mathrm{~nm}$ thick silicon membrane using a spiral scan, $48 \mathrm{pA}$ ion beam current, $0.1 \mathrm{~ms}$ dwell time, and 20 loops. The membrane was suspended over a bulk silicon substrate, in which holes can be seen below the membrane, that are due to intentional over-milling the holes in the membrane. The SEM image was taken at $30^{\circ}$ from the surface normal. The obtained deviation from verticality of the sidewalls is $<1.5^{\circ}$.

Figure 3 shows milling results for a $220 \mathrm{~nm}$ thick silicon membrane. The sidewall angle is much better than in the case of holes in bulk silicon because the milled material can escape both towards the bottom and to the top of the holes, reducing redeposition. In addition the geometry is improved by applying an "over-dose" of about $115 \mathrm{pC}$ per hole, which helps removing redeposited material from the sidewalls. This resulted in a sidewall angle smaller than $1.5^{\circ}$.

\section{ANNEALING}

It has been found that FIB processing of silicon causes strong optical absorption loss in the material. As shown in Fig. 4, the irradiation with gallium ions results in implantation of these ions which can act as electron acceptors in silicon. The figure shows that an extremely high doping level occurs in the top few tens of nanometres, where the silicon is amorphized [17],[18]. Besides, it is well known that tails of the gallium distribution extend hundreds of nanometres into the silicon [19]. The exact mechanism of optical loss caused by gallium-based FIB processing of silicon is not known. It has been found [20] that precipitation of metallic gallium occurs in silicon for the typical milling doses of $\geq 10^{17} \mathrm{~cm}^{-2}$. This may certainly explain the optical loss, but high optical loss has also been found [13] at lower doses where gallium precipitation has not been observed.

As Tanaka et al. [12] have recently shown that a heat treatment significantly lowers optical loss due to FIB damage, and Yokota et al. [21] have shown that gallium escapes from silicon substrates into the atmosphere at elevated temperatures, we did some preliminary experiments to verify that annealing indeed lowers the optical loss, as shown in Fig. 5.

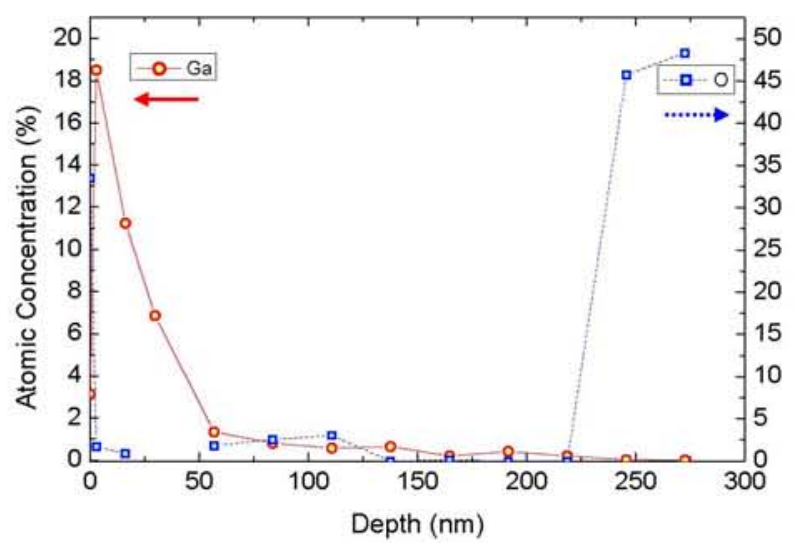

Figure 4. Concentration profiles of gallium (-o) and oxygen (- - ) in a silicon-on-insulator (SOI) layer stack, obtained using X-ray photoelectron spectroscopy (XPS). The irradiation dose was $10^{17}$ ions $/ \mathrm{cm}^{2}$.

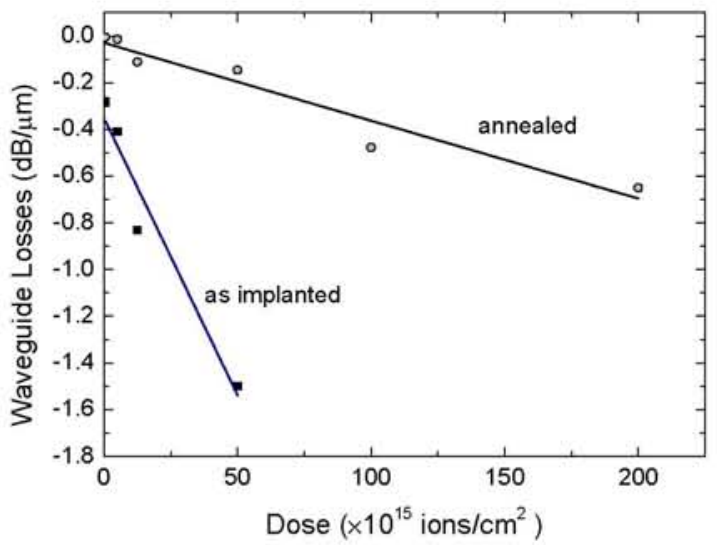

Figure 5. Dose-dependent optical loss measured in silicon photonic wire waveguides having a $600 \mathrm{~nm} \times 200 \mathrm{~nm}$ crosssection. Annealing for 1 hour at $800^{\circ} \mathrm{C}$ led to a significant decrease of the loss. 


\section{CONCLUSIONS}

By choosing a suitable ion beam scanning strategy, the quality of circular holes that are FIB-milled into a silicon substrate can be significantly improved compared to the results obtained by using a linear raster scan. In particular, applying a spiral scan to each individual hole improved the symmetry and the steepness of the sidewalls due to less and better controlled redeposition. FIB-milling of holes in membranes results in much better sidewall verticality since the milled material can more easily escape from the hole, thus reducing redeposition. With some over-milling, the deviation from verticality of the sidewalls of holes in a silicon membrane could be reduced to less than $1.5^{\circ}$. Annealing of waveguides at $800^{\circ} \mathrm{C}$ during one hour significantly reduces optical absorption loss of FIB-irradiated silicon waveguides.

\section{ACKNOWLEDGEMENTS}

We like to thank Vishwas Gadgil for his help with operating the FIB machine and Wenbin Hu for her contribution to the development of scanning routines. The stimulating discussions with Kobus Kuipers and Markus Pollnau are gratefully acknowledged.

\section{REFERENCES}

[1] R.L. Seliger, et al.: A high-intensity scanning ion probe with submicrometer spot size, Appl. Phys. Lett., vol. 34, pp. $310-312,1979$.

[2] A.A. Tseng: Recent developments in nanofabrication using focused ion beams, Small, vol. 1, pp. 924-939, 2005.

[3] A.A. Tseng: Recent developments in micromilling using focused ion beam technology, J. Micromech. Microeng., vol. 14, pp. R15-R34, 2004.

[4] F. Watt, et al.: Ion beam lithography and nanofabrication: a review, Int. J. Nanosci., vol. 4, pp. 269-286, 2005

[5] S. Reyntjens, R. Puers: A review of focused ion beam applications in microsystem technology, J. Micromech. Microeng., vol. 11, pp. 287-300, 2001.

[6] M.J. Cryan, et al:: Focused ion beam-based fabrication of nanostructured photonic devices, IEEE J. Sel. Top. Quantum Electron., vol. 11, pp. 1266-1277, 2005.

[7] M. Roussey, et al.: Electro-optic effect exaltation on lithium niobate photonic crystals due to slow photons, Appl. Phys. Lett. vol. 89, pp. 241110/1-3, 2006.

[8] P. Mühlschlegel, et al.: Resonant optical antennas, Science, vol. 308, pp. 1607-1609, 2005.

[9] C.G. Bostan, et al:: Fast prototyping of planar photonic crystal components using a combination of optical lithography and focused ion beam etching, in Proc. ECIO2005, Grenoble, France, April 2005, pp. 534-537.

[10] K. Balasubramanian, P.J. Heard, M.J. Cryan: Focused ion beam fabrication of two dimensional photonic crystals in silicon-on-insulator, J. Vac. Sci. Technol. B, vol. 24, pp. 2533-2537, 2006.

[11] W.C.L. Hopman, et al: Focused ion beam scan routine, dwell time and dose optimizations for submicrometre period planar photonic crystal components and stamps in silicon, Nanotechnology, vol. 18, pp. 195305/1-11, 2007.

[12] Y. Tanaka, et al: Fabrication of two-dimensional photonic crystal slab point-defect cavity employing local threedimensional structures, Jpn. J. Appl. Phys., vol. 45, pp. 6096-6102, 2006.

[13] J. Schrauwen, D. Van Thourhout, R. Baets: Focused-ion-beam fabricated vertical fiber couplers on silicon-on-insulator waveguides, Appl. Phys. Lett., vol. 89, pp. 141102/1-3, 2006.

[14] J. Schrauwen, et al.: Focused-ion-beam fabrication of slanted fiber couplers in silicon-on-insulator waveguides, in Proc. ECIO2007, Copenhagen, Denmark, April 2007, paper ThB4.

[15] W.C.L. Hopman, et al.: Focused ion beam milling strategies of photonic crystal structures in silicon, in Proc. ECIO2007, Copenhagen, Denmark, April 2007, paper FA2.

[16] S. Kalbitzer: Semiconductors for optical memories, Curr. Opin. Solid State Mater. Sci., vol. 6, pp. 271-279, 2002.

[17] T.L. Matteson, et al.: Electron backscattering diffraction investigation of focused ion beam surfaces, J. Electron. Mater., vol. 31, pp. 33-39, 2002.

[18] B. Basnar, et al.: Focused ion beam induced surface amorphization and sputter processes, J. Vac. Sci. Technol. B, vol. 21, pp. 927-930, 2003

[19] C. Lehrer, et al:: Defects and gallium - contamination during focused ion beam micro machining, in Proc. IEEE Ion Implantation Technol. 2000, pp. 695-698, 2000

[20] L. Frey, C. Lehrer, H. Ryssell: Nanoscale effects in focused ion beam processing, Appl. Phys. A, vol. 76, pp. 1017-1023, 2003.

[21] K. Yokota, et al.: Background impurity dependence of redistributions of implanted gallium in silicon during annealing, J. Appl. Phys., vol. 68, pp. 5385-5387, 1990. 